\title{
Trusted person with elderly patients in France: protocol of a scoping review
}

Luigi Gontard ${ }^{1}$, Maïva Ropaul², Colin Sidre ${ }^{3}$

\begin{abstract}
Background: The background of this study is the recent adoption and updates of surrogate medical decision making in France. The legal designation of a trusted person, meant to ensure that the patient's wishes are made known, has first been introduced in France by the 2002 law on the rights of the sick and the quality of the health system (also known as "Kouchner law"). Some updates came later with the 2005 law relative to patient's rights and end of life («Leonetti law ») and the 2016 law on the rights of persons at the end of life ( Claeys-Leonetti law »). The study addresses the question of the effectiveness and challenges of this system in the French context.
\end{abstract}

Objective: The review was guided by the question: "what is the extent of research in France on patientcentered, family-centered and health professionals-centered outcomes associated with the trusted person system, when elderly patients are involved?". The aims are to measure the extent of the research on surrogate medical decision-making research in France for elderly patients, how well all subpopulations are represented, the methodologies used and whether they are sufficient in describing patient-centered, family-centered and health professionals-centered outcomes associated with surrogate medical decision-making.

Searches: We used 11 scientific databases in order to conduct our research: PubMed, ScienceDirect, EconLit, CINHAL Plus, Embase, LISSA, Cairn.info, the Bibliothèque numérique de droit de la santé et d'éthique médicale (BNDS), the Banque de données en santé publique (BDSP), the Catalogue et index des sites médicaux de langue française (CISMEF) and the Plateforme nationale pour la recherche sur la fin de vie (PNRFV).

Types of study to be included: Quantitative, qualitative, and mixed-method studies were included in order to consider different aspects of research and data collection on surrogate medical decision making.

Design: Online databases were used to identify papers published during the period 2002-2021, from which we have selected publications written in French or English, applying our search strategies.

\footnotetext{
${ }^{1}$ ENS Paris Saclay.

${ }^{2}$ Corresponding author: maiva.ropaul@u-paris.fr . LIRAES (EA 4470), Université de Paris. LIEPP, Fondation Nationale des Sciences Politiques.

${ }^{3}$ Université de Paris.
} 
Flow chart describing the selection process:

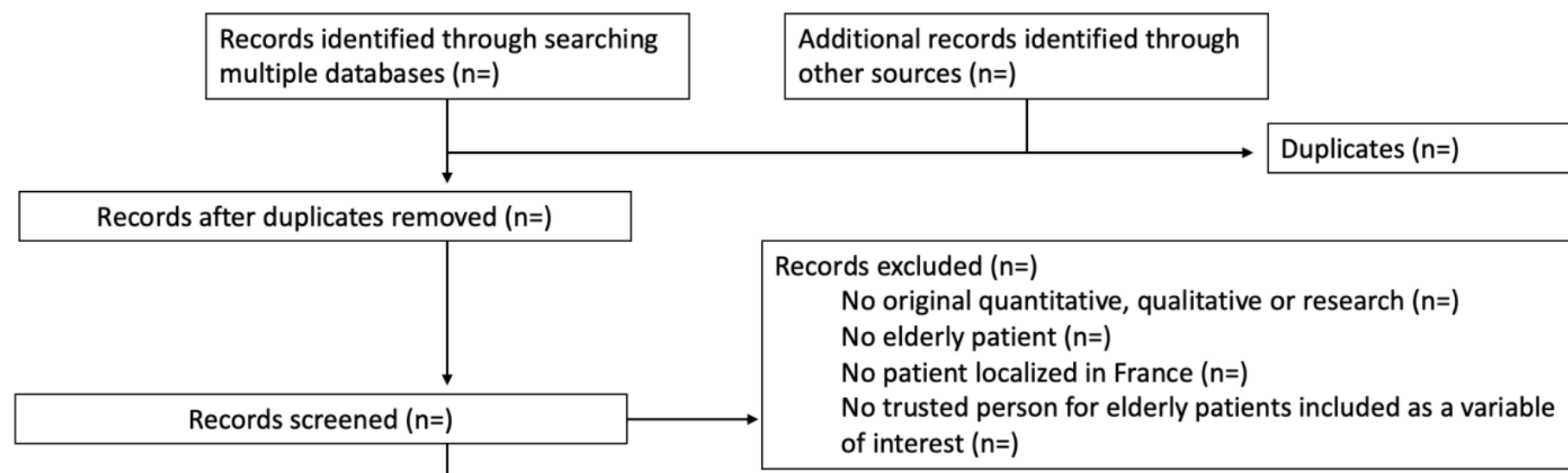

Results: No progress on this item at this time.

Conclusions: No progress on this item at this time.

Keywords: trusted person, surrogate decision maker, medical decision making, elderly patients, France, scoping review.

\section{INTRODUCTION}

Rational: Any adult can appoint a trusted person. This person can be a family member, a close friend or the doctor treating the patient. The trusted person must be designated on a written document co-signed by the patient and the trusted person. It can be modified and revoked at any time. When the patient is unable to express his or her wishes and to receive the medical information necessary to decide, the trusted person is called upon by the medical team. His or her mission is then to give an account of the patient's wishes. His or her testimony prevails over any other testimony. If the patient so wishes, the trusted person can also play a supportive and accompanying role. Thus, he or she can accompany the patient in his or her procedures and attend medical interviews to help him or her make decisions (CSP art. L.1111-6 al. 2). The law (CSP art. L.1111-12) provides that the doctor is required to enquire about the expression of the patient's wishes. If the patient's wishes cannot be ascertained by means of advance directives, the doctor must obtain the testimony of the trusted person or, failing that, any other testimony from the family or close friends.

Elderly people are entering old people's homes at an increasingly advanced age, particularly with the rise of what is known as "home-based care" (Le Bihan, 2012). Furthermore, 150,000 people living in old people's homes died in 2015, which represents a quarter of the deaths recorded throughout France (Muller and Roy, 
2018). These facts raise the question of the consideration of the wishes of elderly patients and the effectiveness of the trustworthy person system.

Moreover, when an elderly person in full possession of his or her cognitive abilities can designate the trusted third party, several questions arise. The trusted third party may be confronted with the problem of the end of life, which may create specific challenges. Nevertheless, the actions of the trusted person can improve the management of the end of life of the elderly to some extent. Thus, understanding the actions of the trusted person, his or her specific challenges and needs can help improve the way society can support vulnerable elderly people and their families.

Objectives: To the best of our knowledge, there is no comprehensive inventory of studies carried out on the trustworthy person system in France. We therefore decided to carry out a scoping review in order to gain an overview of the research on the subject of the dependent person of the frail elderly and to identify the gaps in the literature.

The PPC (Population, concept, context) framework has been used in order to conceptualize the review focus. First, the population covered by the study refers to frail elderly individuals, their trusted person, their family and their health professionals. Second, the notion at the center of the study is the trusted person system. Third, we conduct this research in the French context, characterized by an ageing population, the development of home care and a substantial proportion of deaths in nursing homes.

This study undertakes a review of the literature on trusted persons with elderly patients in France. The review was guided by the question: "what is the extent of research in France on patient-centered, family-centered and health professionals-centered outcomes associated with the trusted person system, when elderly patients are involved?". The aims are to measure the extent of the research on surrogate medical decision-making research in France for elderly patients, how well all subpopulations are represented, the methodologies used and whether they are sufficient in describing patient-centered, family-centered and health profesionnals-centered outcomes associated with surrogate medical decision-making.

\section{METHODS}

Protocol and registration: Our research protocol was drafted using the PRISMA extension for scoping reviews (PRISMA-ScR) checklist and was registered prospectively with the Open Science Framework on 23/07/2021. In addition to the PRISMA checklist, we used other resources related to scoping review from the Joanna Briggs Institute (Khalil, et al., 2021; Peters et al., 2021; Pollock et al., 2021).

Eligibility criteria: To conduct the collect, various eligibility criteria have been chosen. Peer reviewed journal papers and grey literature were included if they were:

- $\quad$ Published between the period of 2002-2021,

- Written in English or French,

- $\quad$ Involved surrogate medical decision maker or families of patients above 60 years old

- Involved surrogate medical decision maker or families of patients living in France

- $\quad$ Focus on patient-centered, family-centered and health professionals-centered outcomes associated with the trusted person system in France, when elderly patients are involved

- Quantitative, qualitative, and mixed-method studies were included in order to consider different aspects of surrogate medical decision making

Papers were excluded if they did not fit into the conceptual framework of the study. 
Information sources: The list of data sources and the search strategy were jointly drafted by the reviewers and an experienced librarian, Colin Sidre (Université de Paris). The search strategies were refined through team discussion.

To identify potentially relevant documents, the following bibliographic databases were searched from 2002 to XX/XX/2021: Pubmed, ScienceDirect, EconLit, CINHAL Plus, Embase, LISSA, Cairn.info. LISSA and Cairn.info are two French databases. LISSA is a search engine referencing scientific articles in French in the field of Health. The database is open access and is likely to provide resources on the French situation. Cairn.info provides francophone publications in social sciences and humanities.

We also conducted our research through two other French resources: the Bibliothèque numérique de droit de la santé et d'éthique médicale (BNDS) and the Banque de données en santé publique (BDSP). BNDS is not a database, but a thematic digital library developed by Editions hospitalières, which focuses on health law. BDSP is no longer updated since 2019 but has a rich archive on a French-speaking perimeter (France, Switzerland, Belgium, Canada and Maghreb) on public health issues.

To consider the grey literature on the subject, we used the Catalogue et index des sites médicaux de langue française (CISMEF) and the Plateforme nationale pour la recherche sur la fin de vie (PNRFV). The aim of CISMEF is to identify and describe resources from institutional and professional sources in the health field that are freely and openly available for consultation. CISMEF allows to search for grey literature in French (France, Switzerland, Belgium, Canada). PNRFV has a directory of end-of-life research projects carried out by researchers listed on their platform.

Search: We illustrate our research strategy using the PubMed database as an example. Indeed, this database is relevant to public health research.

PubMed uses the Medical Subject Headings thesaurus (MeSh). The MeSh is a controlled and hierarchically organized vocabulary produced by the National Library of Medicine. It is used for indexing, cataloging, and searching of biomedical and health-related information. However, many resources are not indexed on PubMed, especially recent articles. In addition, PubMed will search other databases containing articles not intended to be indexed. Therefore, two types of searches are required on PubMed. First, we conduct searches in MeSh (pubmed indexing language). Second, we perform the searches in free language.

After this phase of study of free language research, we used the "Add to Search Builder" tool for PubMed to get the following search equation:

("Proxy"[Mesh] OR "proxy consent"[TW] OR "surrogate designation"[TW] OR "substitute consent"[TW]

OR "medical prox*"[TW] OR "patient agent*"[TW] OR "health care agent*"[TW] OR "health care prox*"[TW] OR "trusted person*"[TW] OR "trusted people"[TW] OR "surrogate decision-making"[TW]

OR "surrogate decision-make*"[TW] OR "substitute decision-making"[TW] OR "substitute decisionmake*"[TW] OR "personne de confiance"[OT] OR "personnes de confiance"[OT])

AND ("Aged"[Mesh] OR "aged"[TW] OR "elder*"[TW] OR "Frailty"[Mesh] OR "frail*"[TW] OR "Palliative care"[Mesh] OR "palliati*"[TW] OR "Terminal care"[Mesh] OR "terminal care"[TW] OR "end of life care"[TW])

AND ("France"[Mesh] OR "France"[TW] OR "French"[TW] OR french[LA] OR France[Affiliation])

Selection of sources of evidence: The search results are exported in Zotero. In Zotero the organization is 
paramount. We therefore named the files using the name of the original database, the date of the search and then the number of results. Third we removed the duplicates using the dedicated function and then merged the documents. Fourth, we export each Zotero file in RIS format. Fifth, the RIS files are imported on Rayyan.ai for subsequent title and abstract relevance screening and data characterization of full articles.

The screenings are conducted independently by two reviewers. The first screening focuses on titles and abstracts. The second will look at the integral text. We resolved disagreements on study selection by consensus and discussion between the reviewers. If the disagreement could not be resolved, a third reviewer was used as an arbitrator in the conflict. Finally, we go through all the references of the sources identified to find additional ones.

Data charting process: After the screening process and the solving of potential conflicts among reviewers, two of them extracted the data using a form. This form has been tested by the team on a sample of XX publications. This process has been followed by discussion and corrections of the form. The test phasis ensures that each member of the reviewer team can extract the same data from a corpus. After this preliminary phasis of the study, the data charting was performed sequentially by both reviewers. Regularly, the reviewer team met and discussed the results and continuously updated the data-charting form in an iterative process.

Data items: The charting table records the key information from the selected publications, which are : author(s), year of publication, origin/country of origin (where the source was published or conducted), aims/purpose, population and sample size within the source of evidence (if applicable), methodology / methods, outcomes and details of these (e.g. how measured) (if applicable), key findings that relate to the scoping review question "what is the extent of research in France on patient-centered, family-centered and health professionals-centered outcomes associated with the trusted person system, when elderly patients are involved?", critical appraisal.

Critical appraisal of individual sources of evidence: The critical appraisal of included sources of evidence was performed using the key questions for the critical appraisal by Fawkes, Ward and Carnes (2015). Information for quality assessment was incorporated into the data extraction form.

Synthesis of results: We have extracted and summarized on Excel all the information that seemed relevant. Subsequently this allowed us to highlight the convergences and divergences between the articles on the commonalities highlighted. That process is still in court. When we identified a systematic review, we counted the number of studies included in the review that might potentially met our inclusion criteria and noted how many studies had been missed by our search.

\section{RESULTS}

Selection of sources of evidence: We used the previous blank flow chart, that we filled with our data, to show how was made the selection of sources of evidence.

Characteristics of sources of evidence: No progress on this item currently

Will be completed with item 14 "charting" of the PRISMA extension for scoping reviews (PRISMA-ScR) checklist when the research will be at a more advanced stage

Critical appraisal within sources of evidence: No progress on this item currently. 
Results of individual sources of evidence: No progress on this item currently.

Synthesis of results: No progress on this item currently

\section{DISCUSSION}

Summary of evidence: No progress on this item currently.

Limitations: No progress on this item currently.

Conclusions: No progress on this item currently.

\section{BIBLIOGRAPHY}

Fawkes, C., Ward, E., \& Carnes, D. (2015). What evidence is good evidence? A masterclass in critical appraisal. International Journal of Osteopathic Medicine, 18(2), 116-129.

Le Bihan, B. (2012). The redefinition of the familialist home care model in France: the complex formalization of care through cash payment. Health and Social Care in the Community (2012) 20(3), 238-246.

Muller, M., \& Roy, D. (2018). L'Ehpad, dernier lieu de vie pour un quart des personnes décédées en France en 2015. Études et résultats, 1094.

\section{FUNDING}

This research was funded by a public grant overseen by the French National Research Agency (ANR) as part of the "Investissements d'Avenir" program LIEPP (ANR-11-LABX-0091, ANR-11-IDEX0005-02) and the Université de Paris IdEx (ANR-18-IDEX-0001)." "in-pi] 